\title{
A Multi-parameter Video Quality Assessment Model Based on 3D Convolutional Neural Network on the Cloud
}

\author{
Xue Li ${ }^{1, *}$, Jiali Qiu ${ }^{2}$ \\ ${ }^{1}$ Xidian University, Xi'an, China \\ ${ }^{2}$ Xi'an Jiaotong University,Xi'an, China \\ * Corresponding author: Xue Li (1029211440@qq.com)
}

Manuscript Revised 28 Aug 2021; Accepted 01 Sep 2021; Published 04 Sep 2021

Academic Editor: Jinchao Chen $\odot$

Abstract: As the rapid development of big data and the artificial intelligence technology, users prefer uploading more and more local files to the cloud server to reduce the pressure of local storage, but when users upload more and more duplicate files, not only wasting the network bandwidth, but also bringing much more inconvenience to the server management, especially images and videos. To solve the problems above, we design a multi-parameter video quality assessment model based on $3 \mathrm{D}$ convolutional neural network in the video deduplication system, we use a method similar to analytic hierarchy process to comprehensively evaluate the impact of packet loss rate, codec, frame rate, bit rate, resolution on video quality, and build a two-stream 3D convolutional neural network from the spatial flow and timing flow to capture the details of video distortion, set the coding layer to remove redundant distortion information. Finally, the LIVE and CSIQ data sets are used for experimental verification, we compare the performance of the proposed scheme with the V-BLIINDS scheme and VIDEO scheme under different packet loss rates. We also use the part of data set to simulate the interaction process between the client and the server, then test the time cost of the scheme. On the whole, the scheme proposed in this paper has a high quality assessment efficiency.

Index Terms: Video quality assessment; 3D CNN; packet loss rate; SRCC; PLCC.

\section{Introduction}

Now we have entered the era of big data, portable shooting devices such as digital cameras and smart phones have spawned a large amount of demand for data storage. Due to the limitation of local storage space, most people prefer to store high-definition pictures and videos on the cloud. When more and more users repeatedly upload multimedia files, it not only seriously wastes network bandwidth, but also leads to the occurrence of data redundancy, brings inconvenience to the daily management of cloud storage. The International Data Center indicates that the amount of digital data in 2020 will reach $44 \mathrm{ZB}$, of which about $75 \%$ of the data is duplicated, and the data redundancy on the cloud server used for backup and storage is more than $90 \%$, detecting and deleting multimedia files has become a very important task.

Data Deduplication [1] allows only a single copy of the file to be kept in the server, and users who store similar files have a link to access this copy without having to store the file repeatedly; at the same time, if the file already exists on the server, the user does not have to upload a copy file of it. Cloud service providers rely on deduplication technology to remove duplicate data, which can reduce bandwidth and storage requirements.

A complete duplicate video detection scheme includes video copy detection technology, video ownership authentication and video quality assessment. Duplicate video detection technology is to compare the client video with the server video to see if there is a video similar to the server. Video ownership authentication is to ensure the user's ownership of video and their ability to recover the stored file from the server; Video quality assessment refers to comparing the quality of similar videos on the client side and the server side, leaving the video with better quality on 
the server side, and removing the duplicate videos by deleting the videos with poor quality. Video deduplication can be divided into server-based deduplication and client-based deduplication according to its execution location. Server-based deduplication involves uploading data from the client to the server and then deleting the duplicate data, which requires a lot of upload bandwidth. Clientbased data duplication is to delete duplicate data before uploads the data, which saves a lot of upload bandwidth and server storage space [2]. Therefore, it is the widely used method of data deduplication. In the interaction between client and server, good quality video files are always saved, and the deletion of poor quality video is very helpful for storage management on the cloud.

Since when the user uploads videos to the server, user datagram protocol adopted is a connectionless and unreliable transmission protocol, the data packet may be lost during the transmission process, or the original video itself has problems such as low resolution, video playback is not smooth and so on, so it is necessary to check the original quality of the video and the transmission process, and evaluate the degree of distortion about video.

At present, the research on video quality assessment on the cloud is still in its infancy. To solve the above actual problem, we propose a method based on 3D convolutional neural network to evaluate the impact of packet loss rate, codec, bit rate, frame rate, and resolution, extract two stream video distortion features of spatial flow and timing flow, the coding layer is used to reduce the redundancy of distortion information. Through the verification on the LIVE and CSIQ data sets, it can be seen that the proposed scheme has a high video quality assessment efficiency.

\section{Related Works}

As we all know, the storage space occupied by video is very large. Compared with other multimedia content, from the network throughput, the requirements of video content are higher, so it is necessary to remove duplicate videos on the cloud server side. To judge the quality of a video, comprehensive consideration should be taken about the number of lost packets during the transmission process, image quality clarity, and fluency [13].

At present, researchers have proposed many methods for evaluating video quality. Common video quality assessment (VQA) $[9,10]$ algorithms can be divided into the following three categories: full-reference (FR), reduce-reference (RR) and noreference (NR) video quality assessment algorithms. The full reference video quality assessment algorithm needs to obtain all the information of the cloud and client videos for similar comparison.
The client's reference video needs to ensure that there is no distortion. The quality assessment is carried out by comparing the difference between the reference video and the video to be evaluated; Reduce-reference (RR) video quality assessment algorithm needs to get part of the information of the reference video and compare it with some features. FR and RR quality assessments take all or part of the reference videos into consideration, such as MSE, PSNR; No-reference (NR) video quality assessment algorithm refers to the perception of video quality without reference video, and often uses the information of the video itself as the quality assessment criteria, such as resolution, color, etc.

In recent years, with the excellent results of deep convolutional neural networks in the field of video feature extraction, researchers are more inclined to apply $3 \mathrm{D}$ convolutional neural networks to video quality assessment. A typical scheme is: $\mathrm{Li}$ et al. [15] proposed an NR-VQA method based on 3D shearlet transform and CNN to effectively extract the anisotropic features of the captured video. Yao et al. [3] designed a bitrate-based metric, which combined the visual perception of video contents and achieved a good generalization property. Valderrama et al. [4] trained the convolutional neural network according to different properties, such as various lengths of the group of pictures (GOP), prioritization policies (BestEffort and DiffServ), altogether with bottlenecks, however, this method used low-resolution images and a limited number of lost packets. Søgaard et al. [5] suggested a regression function for video quality calculation with a correlation coefficient varying from 0.7 to 0.9 based on the video content of dynamic and static scenes, but this method used image evalution indicators to measure the performance. When the spatial information of video is greatly damaged, it will have a greater impact on the test results. Therefore, the choice of video quality evalution indicators, spatial quality assessment and timing information quality assessment, packet loss rate are the indispensable consideration when we evaluate video quality. In addation, $\mathrm{Li}$ et al. [22] proposed a unified NR VQA framework. it contains a mixed datasets training strategy for in-the wild videos, which is based on VSFA. Two kinds of losses are used to deal with the main training of mixed data, namely the loss caused by monotonicity and the loss caused by linearity. Qian et al. [23] developed a novel multiple instance learning (MIL) based model, VQA-MIL, which dynamically adjusts the weights by a block-wise attention module and enriches the features of video bags by a MI Pooling layer. these have great reference significance for video quality evaluation. 
We design a video quality assessment model based on 3D convolutional neural network, which captures video distortion information from two dimensions of space and time, and focuses on evaluating the impact of packet loss rate and codec on video quality, using Spearman's Rank Order Correlation Coefficient (SRCC), Pearson's Linear Correlation Coefficient (PLCC) to evaluate model performance on LIVE and CSIQ data sets, it shows a good advantage both in terms of video quality assessment efficiency and time cost.

\section{Methods}

In this section, we will elaborate the unsupervised quality assessment model based on 3D convolutional neural network from two aspects: the scheme framework and the algorithm details.

\subsection{Scheme Framework}

The scheme framework mainly consists two parts: the first video uploader and the subsequent video uploader. The details are shown in the table below.

\subsection{Algorithm Details}

In this section, we propose a multi-parameter video quality assessment model based on 3D CNN on the cloud. It is obtained by calculating the perceptual distortion value during the change of the video sequence. Since the loss of data packets has a great impact on the measurement of the degree of video distortion, we weight the spatial entropy difference of different influencing factors such as data packet loss, codec, bit rate, frame rate, resolution,

\begin{tabular}{|l|}
\hline \multicolumn{2}{|c|}{ Video quality assessment of the first uploader } \\
\hline Client \\
\hline $\begin{array}{l}\text { Request to upload the quality score of video } \\
\text { quality }(V) \rightarrow V_{Q} \\
\text { Upload the quality score parameter set }\left\{V, V_{Q}\right\}\end{array}$ \\
(1) After performing similarity detection and ownership \\
authentication on the video, if no video similar to the video \\
uploaded by the client is found on the server side, the user is the \\
initial uploader of the video. The server requests the client to upload \\
the quality score parameter of the video; \\
(2)The client calculates the quality score parameter quality $\left.(V) \rightarrow V_{Q}\right\}$ \\
and uploads $\left\{V, V_{Q}\right\}$ to the server; \\
(3)The server stores $\left\{V, V_{Q}\right\}$ in the database.
\end{tabular}

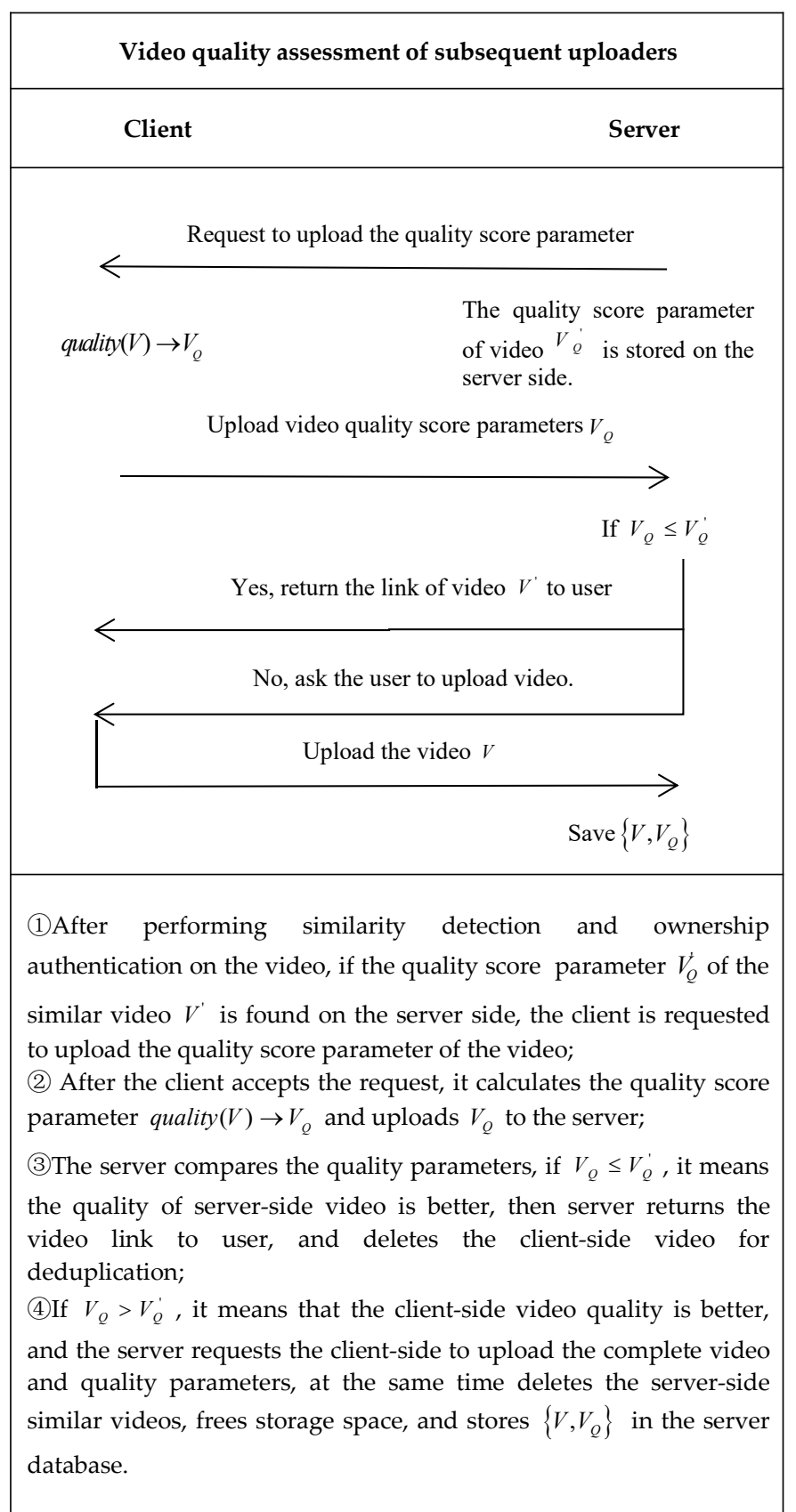

etc., to capture the distortion of the video frame better. The implementation details are as follows:

\subsubsection{Multivariate Assessment}

We use the method similar to analytic hierarchy process to evalute the influence of packet loss rate, codec, bit rate, frame rate, and resolution on video quality. The packet loss rate and the codec are two very important factors that affect the video quality [7]. Packet loss refers to the situation where the data packet has server bits error during the transmission process or the transmission delay exceeds a set threshold, which causes multiple data packets fail to reach the destination, packet loss will seriously affect the smoothness of the video. Most of the application of codec cause damage to the video quality, because the compression process is at the expense of video quality, such as MPEG-2 and H. 264 codec 
and so on. In addition, the bit rate, frame rate, and resolution also affect the video quality. The bit rate refers to the number of data bits transmitted per unit time. The bit rate is higher, the video is clearer. Frame rate refers to the number of frames transmitted in a unit time, the frame rate is larger, the video is smoother. Resolution refers to the size of the video frame, the resolution is higher, the video frame is larger. We take the best video quality as the target layer, take the packet loss rate, codec, bit rate, frame rate, and resolution as criterion layer, compare the influence of the five factors at the criterion layer on the target layer [11].

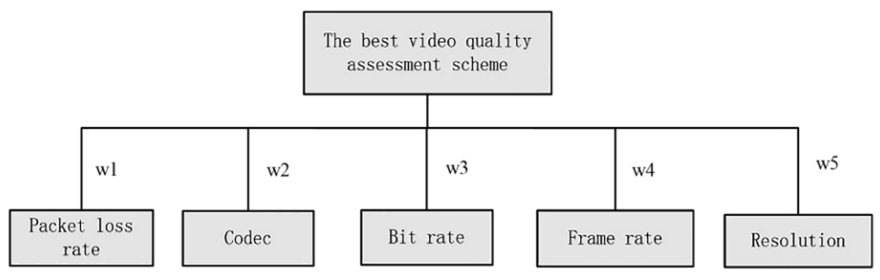

Fig. 1. The Video quality assessment scheme hierarchy figure

According to the degree of influence about each two factors on the target layer, a paired comparison matrix is constructed. The Table 1 shows the meaning of the 1-9 comparison scale in the paired comparison method, where: $\left\{C_{1}, C_{2}, C_{3}, C_{4}, C_{5}\right\}=$ $\{$ packet loss rate, codec, bit rate, frame rate, resolution $\}, i, j \in$ $\{1,2,3,4,5\}$.

Table 1. The meaning of the 1-9 comparison scale.

\begin{tabular}{ll}
\hline Scale $a_{i j}$ & Meaning \\
\hline 1 & $C_{i}$ and $C_{j}$ have the same effect \\
3 & The effect of $C_{i}$ is slightly stronger than $C_{j}$ \\
5 & The effect of $C_{i}$ is stronger than $C_{j}$ \\
7 & The effect of $C_{i}$ is obviously stronger than $C_{j}$ \\
9 & The effect of $C_{i}$ is absolutely stronger than $C_{j}$ \\
$2,4,6,8$ & The ratio of the influence of $C_{i}$ to $C_{j}$ is between the \\
& above two adjacent levels \\
$1,1 / 2, \ldots, 1 / 9$ & $\begin{array}{l}\text { The ratio of the influence of } C_{i} \text { to } C_{j} \text { is the reciprocal } \\
\text { number of } a_{i j}\end{array}$ \\
\hline
\end{tabular}

Through the multiple comparisons, the pairwise comparison matrix of the criterion layer to the target layer is obtained as:

$$
A=\left[\begin{array}{ccccc}
1 & 5 & 6 & 8 & 9 \\
\frac{1}{5} & 1 & 4 & 6 & 8 \\
\frac{1}{6} & \frac{1}{4} & 1 & 3 & 3 \\
\frac{1}{8} & \frac{1}{6} & \frac{1}{3} & 1 & 2 \\
\frac{1}{9} & \frac{1}{8} & \frac{1}{3} & \frac{1}{2} & 1
\end{array}\right]
$$

Calculate the largest eigenvalue of matrix $A$ and the corresponding eigenvector, the maximum eigenvalue can be obtained as $\lambda=5.3443$.

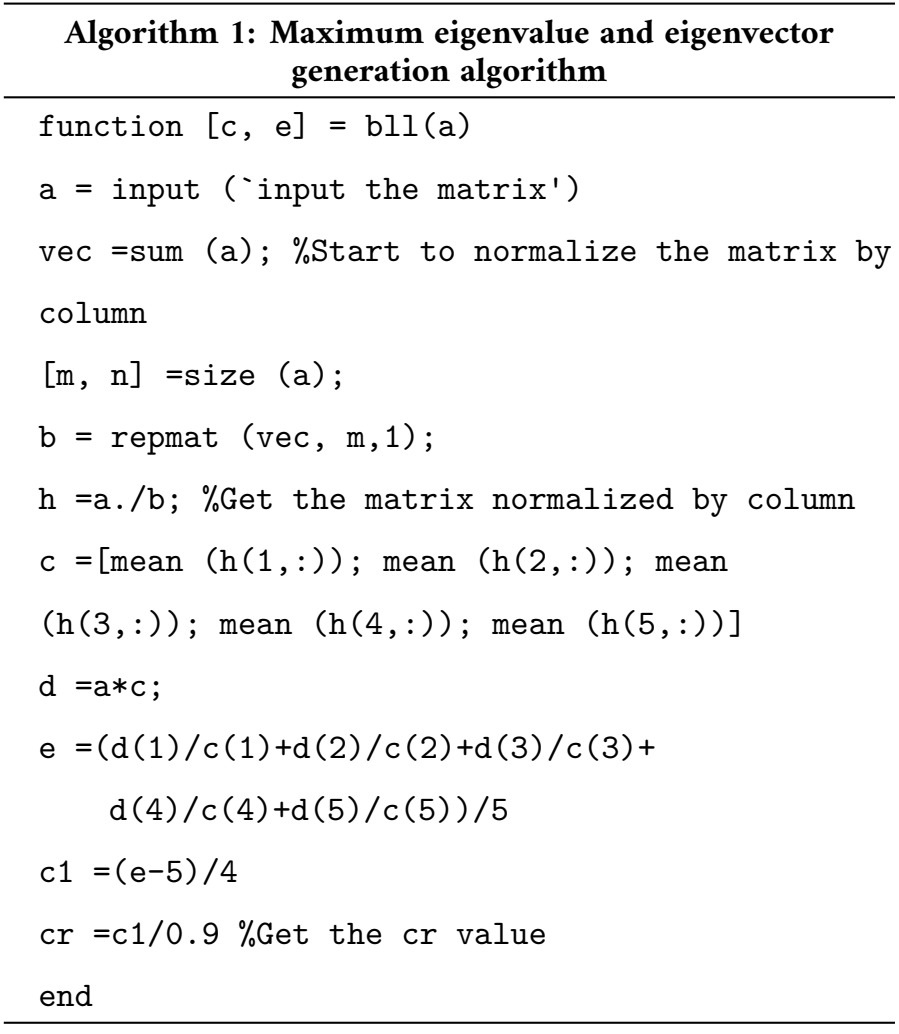

Then check the consistency of matrix $A, C I$ can be calculated according to the following formula:

$$
C I=\frac{\lambda-n}{n-1}
$$

When $\lambda=5.3443, n=5$, we can get $C I=0.0861$, and refer to the numerical table of random consistency indicators to get the random consistency index corresponding to matrix $\mathrm{A}$ is $R I=1.12$. According to the consistency index and the random consistency index, we can obtain the consistency ratio:

$$
C R=\frac{C I}{R I}=0.077
$$

Because $C R<0.1$, so the degree of inconsistency of matrix $A$ is within the allowable range. Therefore, the feature vector $w=$ $(0.5453,0.2585,0.1041,0.0546,0.0375)^{T}$ can be considered as the measure of the degree of influence of each factor.

Table 2. Random consistency index numerical table.

\begin{tabular}{lllllll}
\hline$n$ & $\mathbf{1}$ & $\mathbf{2}$ & $\mathbf{3}$ & $\mathbf{4}$ & $\mathbf{5}$ & $\mathbf{6}$ \\
\hline$R I$ & 0 & 0 & 0.58 & 0.90 & 1.12 & 1.24 \\
$n$ & 7 & $\mathbf{8}$ & $\mathbf{9}$ & $\mathbf{1 0}$ & $\mathbf{1 1}$ & \\
$R I$ & 1.32 & 1.41 & 1.45 & 1.49 & 1.51 & \\
\hline
\end{tabular}

\subsubsection{D CNN Model Construction}

In this section, based on the influence factor weights obtained in the previous section, we construct a two-stream 3D convolutional neural network distortion model. The specific implementation details are as follows: 
A: Extend the 2D convolutional network to $3 \mathrm{D}$. The threedimensional expansion of the network is achieved by expanding all filters and pooling kernels, that is, giving the $2 \mathrm{D}$ network an extra time dimension, turning the filter $N \times N$ into $N \times N \times$ $N$. Due to the existence of the linear relationship between video sequences, the filter weights in $2 \mathrm{D}$ can be repeated $\mathrm{N}$ times in the time dimension, and then divided by $\mathrm{N}$, which guarantees the same dimension of the convolution filter to a certain extent. Since the output of the video convolutional layer composed of frames is constant in the time dimension, the average layer and the maximum pooling layer are both consistent with the $2 \mathrm{D}$ network structure $[20]$.

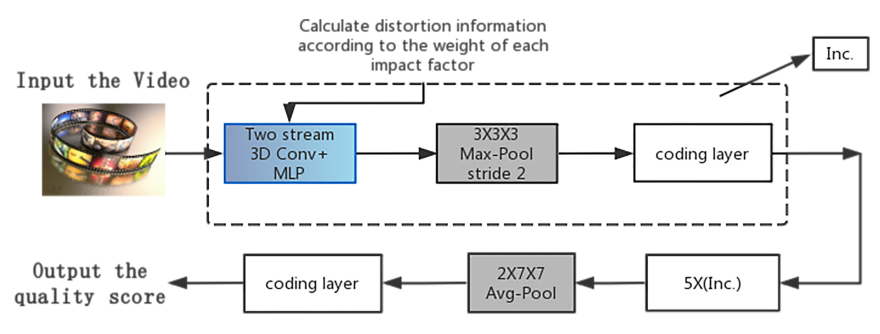

Fig. 2. The network structure of MQA

B: Consider the growth rate of space, time and network depth. For all frames in the video stream, their spatial structure (horizontal and vertical) is the same, so their pooling kernel and step size should also be the same, which means that the deeper information is in the spatial dimension are affected equally by the position of frames that are getting farther and farther away, but due to the existence of time factors, receiving under the same conditions does not meet the expected results, because there will be the effect of the frame rate and the image dimension. If compared to the space domain, the time domain increases too fast, the more fine-grained distortion information is difficult to capture. If the increase in time is too slow, it is easy to misjudge the degree of distortion of the video.

C: Two-stream 3D CNN structure. Although the 3D convolutional neural network can learn distortion information directly from the input continuous video frames, it still performs pure feedforward calculations, and the optical flow algorithm is periodic in a sense (For example, it can iteratively optimize the optical flow field). Therefore, the idea of two-stream structure should be adopted. One stream only receives the RGB stream distortion information, and the other stream only receives the optimized smooth optical flow distortion information, and then calculate the average of the two results.

D: Both spatial distortion information and timing distortion information are calculated using 3D CNN and the feedforward neural network MLP, to calculate the degree of deviation between the actual output of the network and the expected output, then get the quality score of video [24].

Multilayer Perceptron (MLP) is a feedforward neural network consisting of an input layer, at least one hidden layer, and an output layer. In practical applications, in order to solve more complex problems, we generally choose an activation function to provide better adaptability for classification. MLP is widely used for prediction, classification, and recognition. It can solve nonlinear separable problems, perform iterative learning, load data sets into the network one by one at a time, and change the weights associated with the input values each time.

We use $w=(0.5453,0.2585,0.1041,0.0546,0.0375)^{T}$ as the weight of the distortion information extraction of the two-stream $3 \mathrm{D} \mathrm{CNN}$, use the delta rule to update the input weight and minimize the error in the output of the neural network through gradient descent. The error value of a single output neuron is a function of its actual value and target value. The total error of the network is the sum of all error values from all output neurons.

$$
\text { Error }_{\text {total }}=\sum_{i=1}^{n} \frac{1}{2}\left(\text { tar }_{i}-a c t_{i}\right)^{2}
$$

E: During the training period, we set the rectified linear unitReLU as the activation function, and test the performance of the activation function based on the measured values SRCC and PLCC. For the detail information about SRCC and PLCC, please see section 4.2. Each activation function is gradually implemented into the hidden layer, we set 100 nodes for per hidden layer, and the accuracy is calculated according to the closeness of the actual model output to the target output. The purpose of using the hidden layer is to abstract the degree of distortion of each influencing factor better.

F: Define an encoding layer to extract meaningful errors from each layer of the deep CNN model, and remove the redundant information through the encoding layer, finally connect these errors as a distortion score.

G: Use dropout and k-fold cross-validation methods to prevent overfitting, and set the learning rate $I=0.00163$. Train the model 100 times, and put 800 videos into each training.

$\mathrm{H}$ : Construct the loss function to train the model:

$$
\text { Loss }=\frac{\sum_{i=1}^{n}\left\|f\left(x_{i}, w\right)-y_{i}\right\|}{n}
$$

Where $n$ is the num of videos, $w$ represents a parameter in the network, $y_{i}$ represents the tagged score of the video, $f\left(x_{i}, w\right)$ represents the predicted score of the network. 
I: In each convolutional layer calculated by convolutional neural network, weight calculation will be carried out for influencing factors of video quality-data packet loss, frame rate, bit rate and resolution.

\section{Experiments}

The simulation experiment environment in this section is as follows:

The processor is Inter (R) Core (TM) i5-8265U CPU@ $1.6 \mathrm{GHz}$, the graphics card is NVIDIA GeForce MX230, the memory size is $8 \mathrm{G}$, the operating system is Ubuntu 16.04 , and the development platform is TensorFlow 1.14.0 and matlab R2016a. Due to hardware constraints, the server side of this article adopts the same configuration as the client side.

\subsection{Datasets}

We used the data set LIVE [6] and CSIQ to verify the performance of the model, there are 160 videos in the data set LIVE, including 10 lossless videos, and each lossless video corresponds to 15 videos with different distortion types and degrees. There are four types of distortion, MPEG-2 compression distortion, $\mathrm{H}$. 264 compression distortion, IP network distortion and infinite network distortion. Each video has a subjective score, which is marked by dozens of subjects. The score range is $0-100$, the higher the score is, the worse the quality of the video is.

The Subjective Video Quality Database (CSIQ) contains 228 videos, of which 12 are original videos and 216 are distorted videos, All videos in the database are in YUV420 format with a resolution of $832 \times 480$ pixels, a duration of 10 seconds, and a frame rate range of $24,25,30,50$, and 60 FPS. The distortion types include four compression based distortion types and two transmission based distortion types: H. 264 compression (h. 264), HEVC/h. 265 compression (HEVC), Dynamic JPEG compression (MJPEG), Wavelet-based compression using the SNOW codec (SNOW), H. 264 video suffers analog WIRELESS transmission loss (Wireless) and Additive White Noise (AWGN). In the paper, to prevent overfitting, the data sets were divided into training set, test set and cross-validation set, the ratio is $70: 15: 15$. We used LIVE and CSIQ data sets to create a total of 32079 distortion samples for training, verifying, and testing neural networks. The validation set was mainly used to confirm the ability of the neural network to predict the user's perceived quality. The training and verification process was repeated 10 times, and 10-fold cross-validation was performed to make the average accuracy score more robust.

\subsection{Evaluation Index}

In order to evaluate the performance of the proposed video quality algorithm, Spearman's Rank Order Correlation Coefficient (SRCC) and Pearson's Linear Correlation Coefficient (PLCC) are used to measure the correlation between a set of estimated visual quality scores and the human subjective quality score, it means:

$$
\left\{\begin{array}{l}
\operatorname{SRCC}\left(Q_{\mathrm{est}}, Q_{\mathrm{sub}}\right)=1-\frac{6 \sum d_{i}}{m\left(m^{2}-1\right)} \\
\operatorname{PLCC}\left(Q_{\mathrm{est}}, Q_{\mathrm{sub}}\right)=\frac{\operatorname{cov}\left(Q_{\mathrm{est}}, Q_{\mathrm{sub}}\right)}{\sigma\left(Q_{\mathrm{est}}\right) \sigma\left(Q_{\mathrm{sub}}\right)}
\end{array}\right.
$$

Where $m$ is the number of videos in the database, $d_{i}$ is the rank difference of the evaluation sample with serial number $i$ in the two evaluation scores. For the two measurement schemes, when the value is closer to 1 , the measurement performance is better. $P L C C$ measures the degree of linear correlation between videos, $S R C C$ evaluates the predictive monotony of the algorithm.

\subsection{Performance Test}

We first used MQA scheme to evaluate each category of subdistortion in the two data sets, and then carried out the overall video quality assessment, and compared the performance with the video quality assessment algorithms of V-BLIINDS and VIDEO. In order to prevent the experimental results from being accidental, the calculation process was repeated for 10 times in each time. The results obtained are shown in the table below.

Table 3. Quality assessment result table of LIVE data set.

\begin{tabular}{lllllll}
\hline Indicat-ors & Schemes & MPEG-2 & H.264 & IP & Wireless & Total \\
\hline \multirow{2}{*}{ SRCC } & V-BLIINDS & 0.620 & 0.749 & 0.692 & 0.873 & 0.718 \\
& VIDEO & 0.801 & 0.775 & 0.711 & 0.814 & 0.805 \\
& MQA & 0.972 & 0.930 & 0.994 & 0.869 & 0.914 \\
\multirow{2}{*}{ PLCC } & V-BLIINDS & 0.781 & 0.709 & 0.644 & 0.851 & 0.733 \\
& VIDEO & 0.802 & 0.722 & 0.681 & 0.899 & 0.819 \\
& MQA & 0.953 & 0.935 & 0.930 & 0.972 & 0.966 \\
\hline
\end{tabular}

Analysis of the above tables show that when the SRCC and PLCC coefficients are used to quantify the video quality, the closer the two coefficient values are to 1 , the better the visual fitting effect to the human eye. It can be seen that the SRCC and PLCC coefficients of the scheme proposed in this article are almost all larger than the V-BLIINDS scheme and the VIDEO scheme, and most are above 0.9. But on the data set LIVE, for wireless network distortion, when SRCC coefficients are used for evaluation, the MQA scheme is slightly lower than the V-BLIINDS scheme. It may be that when this article evaluates the influence of each factor, the calculated weights do not describe the type of wireless 
Table 4. Quality assessment result table of CSIQ data set.

\begin{tabular}{llllll}
\hline Indicators & Schemes & H.264 & Hevc & Mjpeg & Snow \\
\hline \multirow{2}{*}{ SRCC } & V-BLIINDS & 0.625 & 0.533 & 0.650 & 0.712 \\
& VIDEO & 0.758 & 0.710 & 0.629 & 0.883 \\
& MQA & 0.953 & 0.970 & 0.939 & 0.963 \\
\hline \multirow{2}{*}{ Indicators } & Schemes & Wireless & Awgn & Total & \\
\hline \multirow{2}{*}{ Indicators } & V-BLIINDS & 0.648 & 0.715 & 0.694 & \\
& VIDEO & 0.873 & 0.749 & 0.817 & \\
& MQA & 0.971 & 0.943 & 0.969 & \\
\hline \multirow{2}{*}{ PLCC } & V-BLIINDS & 0.649 & 0.746 & 0.592 & 0.798 \\
& VIDEO & 0.735 & 0.830 & 0.591 & 0.622 \\
& MQA & 0.956 & 0.972 & 0.959 & 0.982 \\
\hline Indicators & Schemes & Wireless & Awgn & Total & \\
\hline \multirow{2}{*}{ PLCC } & V-BLIINDS & 0.705 & 0.897 & 0.731 & \\
& VIDEO & 0.873 & 0.749 & 0.815 & \\
& MQA & 0.977 & 0.926 & 0.974 & \\
\hline
\end{tabular}

(b)

network distortion well. Figure 3 describes the overall video quality evaluation comparison of the MQA scheme, the V-BLIINDS scheme, and the VIDEO scheme, the two index parameter values of the MQA scheme are above 0.9. In summary, whether it is evaluated from two dimensions of prediction accuracy or monotonicity, whether it is on each distortion subset or on the overall data set, the MQA scheme proposed in this paper can show relatively excellent performance.

In addition, we set different packet loss rates for the 12 original videos in the CSIQ data set and built a distorted video set. The set contains 12 groups, and each group contains 6 distorted videos, corresponding to 6 packet loss rate settings. We calculated the SRCC and PLCC for each packet loss rate in the 12 groups and took the average value. To prevent accidental situations, the calculation results were repeated 10 times and the average value was taken to test the changes in video quality under different packet loss rates.

From the Fig. 4, it can be seen that with the gradual increase of the packet loss rate, the SRCC and PLCC levels of the three schemes all show a downward trend, and the change of MQA scheme tends to be stable and is closest to 1. But for the VIDEO scheme and V-BLIINDS scheme, with the gradual increase of the packet loss rate, the SRCC and PLCC indicators show a trend of slow decline first and then a sharp decline. It indicates that in the case of packet loss distortion, the MQA scheme plays a better video quality assessment role than the other two schemes.

We used videos in the LIVE data set to test the performance of the model, took 10 lossless videos as the videos stored on the

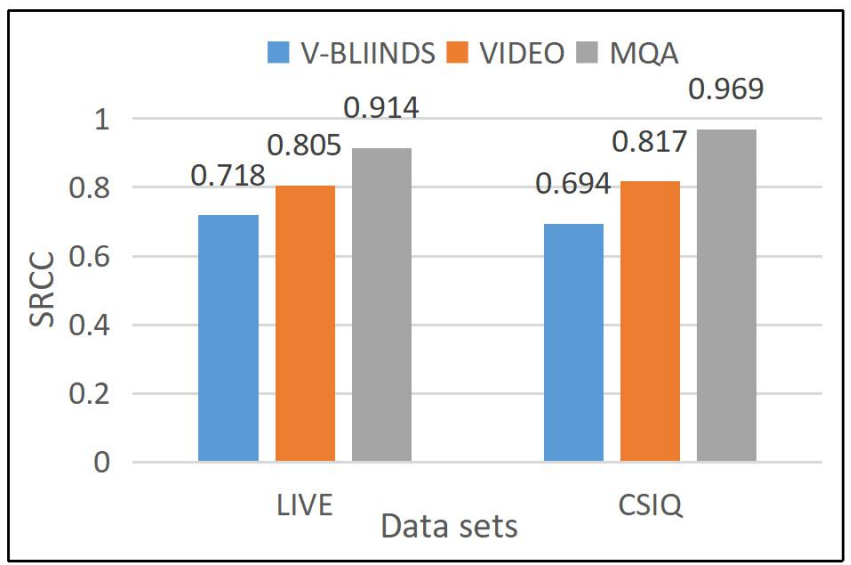

(a)

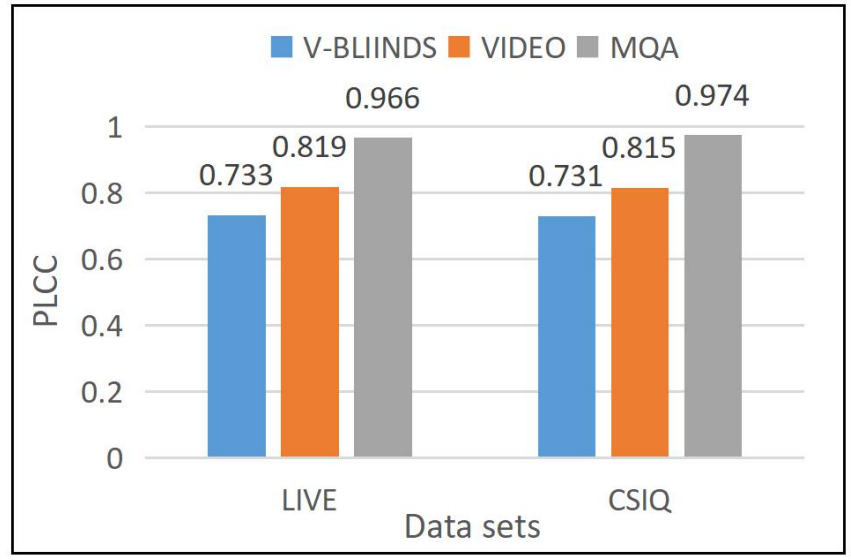

(b)

Fig. 3. Performance comparison of various schemes under SRCC and PLCC evaluation indicators

cloud server, and selected all distorted videos corresponding to the 10 lossless videos as client videos to construct 10 test groups. Calculated the quality score of each group about lossless videos and distorted videos, and used SRCC and PLCC as evaluation indicators. The SRCC value and PLCC value calculated for all distorted videos were averaged as the final result of the client SRCC and PLCC values in Table 5. When the SRCC and PLCC parameters of the server-side video were larger, the quality assessment model can be considered effective. As shown in the following table, we can know that all test groups meet the conditions.

Table 5. Client and server video quality assessment.

\begin{tabular}{lllllll}
\hline \multirow{2}{*}{ Groups } & \multicolumn{2}{c}{ Server } & & \multicolumn{2}{c}{ Client } & \multirow{2}{*}{ If $Q_{S} \geqslant Q_{C}$} \\
\cline { 2 - 3 } & SRCC & PLCC & & SRCC & PLCC & \\
\hline 1 & 0.953 & 0.977 & 0.949 & 0.958 & yes \\
2 & 0.916 & 0.928 & 0.903 & 0.925 & yes \\
3 & 0.947 & 0.951 & 0.943 & 0.949 & yes \\
4 & 0.972 & 0.988 & 0.961 & 0.960 & yes \\
5 & 0.949 & 0.967 & 0.938 & 0.954 & yes \\
6 & 0.991 & 0.989 & 0.985 & 0.987 & yes \\
7 & 0.962 & 0.984 & 0.953 & 0.970 & yes \\
8 & 0.979 & 0.951 & 0.967 & 0.949 & yes \\
9 & 0.984 & 0.935 & 0.971 & 0.903 & yes \\
10 & 0.993 & 0.976 & 0.990 & 0.962 & yes \\
\hline
\end{tabular}




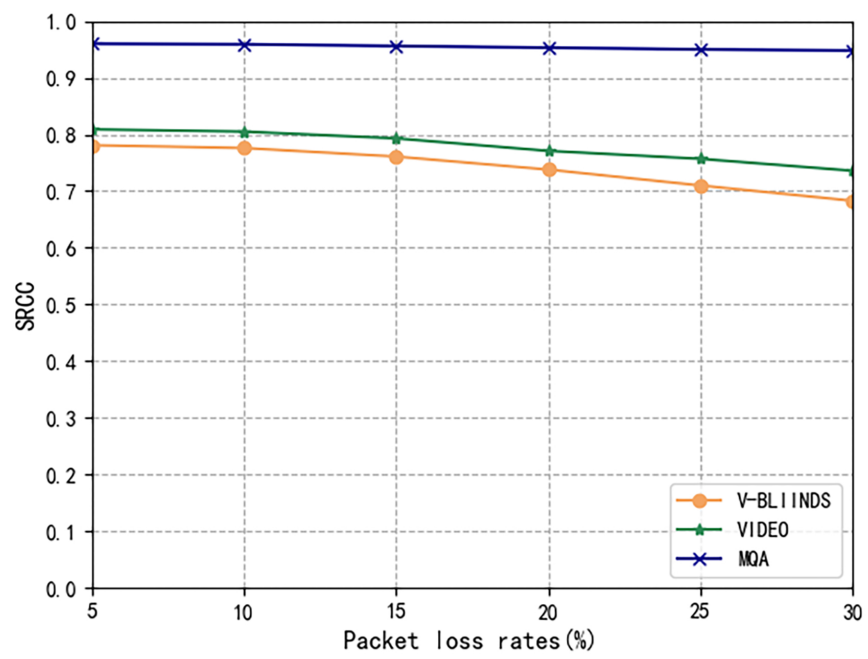

(a)

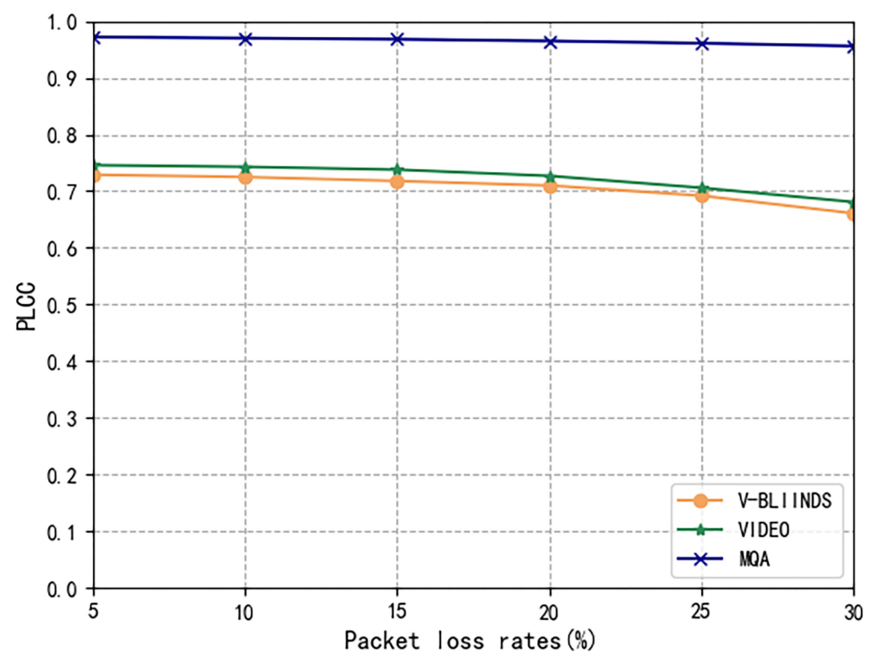

(b)

Fig. 4. Performance comparison of different packet loss rates under SRCC and PLCC evaluation indicators

\subsection{Time Cost}

We still used the videos of the LIVE data set in the above performance experiment to test the model time overhead. The serverside video and the video that needed to be uploaded by the client remain unchanged. The calculation time of the server-side and the client-side in the 10 groups were calculated respectively, the calculation time of all distorted videos on the client side was averaged as the final result, it did not include model training time. Then compared with the total time cost of the V-BLIINDS and VIDEO schemes. The results are shown in the following table. The MQA scheme proposed in this article has a smaller time overhead than the other two schemes. The MQA scheme can save $81.79 \%$ of the average time cost compared with the V-BLIINDS scheme and $22.6 \%$ compared with the VIDEO scheme. The time in this paper was tested on the CPU. If we use the GPU, then will get a smaller time overhead.
Table 6. The time cost of MQA.

\begin{tabular}{llll}
\hline Groups & Time cost of Server $(\mathbf{s})$ & Time cost of Client $(\mathbf{s})$ & Total (s) \\
\hline 1 & 49 & 66 & 115 \\
2 & 65 & 68 & 133 \\
3 & 42 & 46 & 88 \\
4 & 45 & 49 & 94 \\
5 & 60 & 66 & 126 \\
6 & 51 & 58 & 109 \\
7 & 69 & 74 & 143 \\
8 & 47 & 50 & 97 \\
9 & 51 & 54 & 105 \\
10 & 44 & 49 & 93 \\
\hline
\end{tabular}

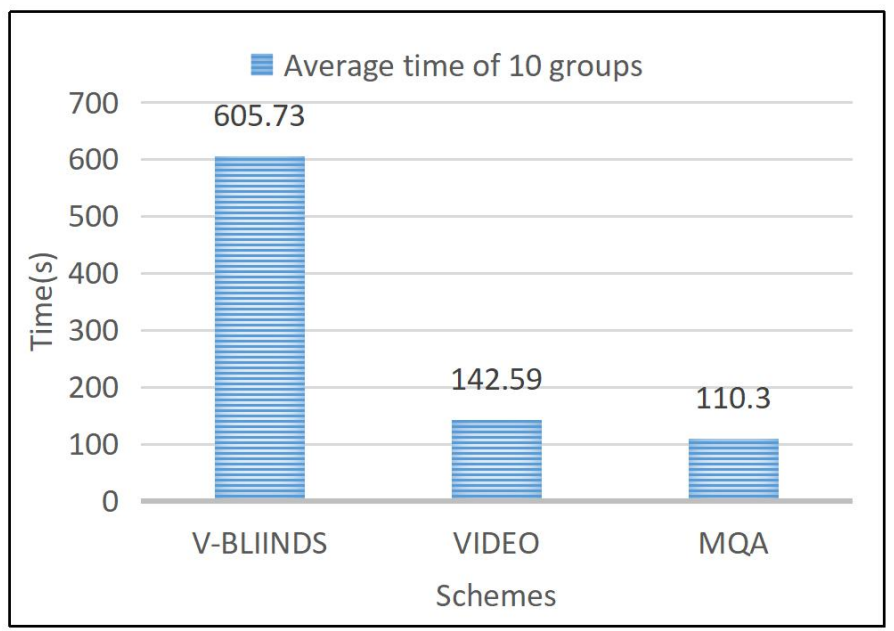

Fig. 5. Comparison of the time cost of the three schemes

\section{Conclusions and Discussions}

In this paper, we propose an effective video quality assessment model, and build an interactive framework for quality assessment between the client and the server. Firstly, we use the method similar to analytic hierarchy process to estimate the impact of packet loss rate, codec, frame rate, bit rate, and resolution on video quality, this is achieved by calculating the weight vector; Secondly, we capture the details of video distortion based on 3D convolutional neural network from the spatial flow and timing flow, define the coding layer to remove the redundant distortion information, use dropout and k-fold cross-validation methods to prevent overfitting, construct a loss function to train the network, and output the video quality score; Thirdly, we use the Spearman's Rank Correlation Coefficient (SRCC) and Pearson's Linear Correlation Coefficient (PLCC) for model assessment, and verify it on the LIVE and CSIQ data sets. According to the experimental results, it can be seen that the scheme proposed in this article is more effective than the V-BLIINDS scheme and the VIDEO scheme to measure video quality. In addition, we also test the evaluation performance about the three schemes under different packet loss rate settings, and part of the data set is used to simulate the interactive pro- 
cess of quality assessment between the client and the server, the test results of all videos are consistent with the preset results; $\mathrm{Fi}$ nally, we use part of the data set to test the time overhead of the MQA scheme, without considering the model training time, it saves more time than the V-BLIINDS scheme and the VIDEO scheme.

The video quality assessment scheme proposed in this article can effectively evaluate the quality of the video from the client and cloud server, but there are still two potential research directions: On the one hand, for the non-uniformly distorted video, the degree of distortion about small video frame blocks are different from each other, so the video distortion information should be captured in a more fine-grained manner with fixed-size blocks as the unit; On the other hand, by constructing supervision information, to enhance the learning ability of network in the case of small labeled data, and increase the adaptability of the network. In the future, we will devote ourselves to the research of the above two aspects

\section{References}

[1] Yang X, Lu R, Choo K K R, et al. Achieving Efficient and Privacy-Preserving Cross-Domain Big Data Deduplication in Cloud[J]. IEEE Transactions on Big Data, 2017: 1-1.

[2] Wu X, Hauptmann A G, Ngo C W. Practical elimination of near-duplicates from web video search $[\mathrm{C}]$. Proceedings of the 15th ACM international conference on Multimedia. ACM, 2007: 218-227.

[3] J. Y. Yao and G. Liu, "Bitrate-based no-reference video quality assessment combining the visual perception of video contents," IEEE Trans. Broadcast., vol. 65 , no. 3 , pp. 546-557, Sep. 2019.

[4] D. Valderrama and N. Gómez, "Nonintrusive method based on neural networks for video quality of experience assessment," Advances in Multimedia, Vol. 2016, 2016.

[5] J. Søgaard, S. Forchhammer and J. Korhonen, Video quality assessment and machine learning: Performance and interpretability, in 7 th International Workshop on Quality of Multimedia Experience (QoMEX), 2015.

[6] Seshadrinathan K, Soundararajan R, Bovik A C, et al. A subjective study to evalute video quality assessment algorithms[C]. //ISesT/SPIE Electronic Imaging. San Jose: IEEE, 2010: 75270H.

[7] Saad M A, Bovik A C, Charrier C. Blind prediction of natural video quality [J]. IEEE Transactions on image Processing, 2014, 23(3): 1352-1365.

[8] Mittal A, Soundararajan R, Bovik A C. Making a completely blind image quality analyzer [J]. IEEE Signal processing Letters, 2013, 22 (3): 209-212.

[9] W. Loh and D. B. L. Bong, A Just Noticeable Difference-Based Video Quality Assessment Method with Low Computational Complexity, Sensing and Imaging, Vol. 19, Article number: 33, 2018. doi: 10.1007/s11220-018-0216-9.

[10] Z. Cheng, L. Ding, W. Huang, F. Yang and L. Qian, A unified QoE prediction framework for HEVC encoded video streaming over wireless networks, in IEEE International Symposium on Broadband Multimedia Systems and Broadcasting (BMSB), Cagliari, 2017, pp. 1-6.

[11] L. Anegekuh, L. Sun, E. Jammeh, I. Mkwawa and E. Ifeachor, Content-based video quality prediction for HEVC encoded videos streamed over packet networks, In IEEE Transactions on Multimedia 17(8) (2015), 1323-1334.

[12] M. Alreshoodi, A. O. Adeyemi-Ejeye, J. Woods and S. D. Walker, Fuzzy logic inference system-based hybrid quality prediction model for wireless $4 \mathrm{k}$ UHD H.265-coded video streaming, In IET Networks 4(6) (2015), 296-303.
[13] Y. Zhang, X. Gao, L. He, W. Lu and R. He, "Blind video quality assessment with weakly supervised learning and resampling strategy," IEEE Trans. Circuits Syst. Video Technol., vol. 29, no. 8, pp. 2244-2255, Aug. 2019

[14] J. F. B. Valderrama and D. J. L. Valderrama, On LAMDA clustering method based on typicality degree and intuitionistic fuzzy sets, Expert Systems with Applications. 107 (2018), 196-221. doi:10.1016/j.eswa.2018.04.022.

[15] Y. Li et al., "No-reference video quality assessment with 3D shearlet transform and convolutional neural networks," IEEE Trans. Circuits Syst. Video Technol., vol. 26, no. 6, pp. 1044-1057, Jun. 2016.

[16] M. Agarla, L. Celona and R. Schettini, "No-reference quality assessment of in-capture distorted videos," J. Imag., vol. 6, no. 8, 2020.

[17] J. Nightingale, P. Salva-Garcia, J. M. A. Calero and Q. Wang, 5GQoE: QoE modelling for Ultra-HD video streaming in 5G networks, IEEE Transactions on Broadcasting 64(2) (2018), 621-634. doi: 10.1109/TBC.2018.2816786.

[18] M. Narwaria and W. Lin, Machine Learning Based Modeling of Spatial and Temporal Factors for Video Quality Assessment, in: Proc. 18th IEEE International Conference on Image Processing (ICIP), 2011, pp. 2513-2516.

[19] P. Debajyoti and V. Vajirasak, A No-Reference Modular Video Quality Prediction Model for H.265/HEVC and VP9 Codecs on a Mobile Device, Advances in Multimedia, Vol. 2017, Article ID 8317590, 2017. doi: $10.1155 / 2017 / 8317590$.

[20] L. Qian et al., "No-Reference Nonuniform Distorted Video Quality Assessment Based on Deep Multiple Instance Learning," in IEEE MultiMedia, vol. 28, no. 1, pp. 28-37, 1 Jan.-March 2021, doi: 10.1109/MMUL.2020.3034338.

[21] Chen, P. ; Li, L. ; Ma, L. ; Wu, J. ; Shi, G. RIRNet: Recurrent-In-Recurrent Network for Video Quality Assessment. In Proceedings of the ACM International Conference on Multimedia, Dublin, Ireland, 26-29 October 2020; pp. 834-842. 28.

[22] Li, D. ; Jiang, T. ; Jiang, M. Unified Quality Assessment of in-the-Wild Videos with Mixed Datasets Training. Int. J. Comput. Vis. 2021. [CrossRef].

[23] L. Qian et al., "No-Reference Nonuniform Distorted Video Quality Assessment Based on Deep Multiple Instance Learning," in IEEE MultiMedia, vol. 28, no. 1, pp. 28-37, 1 Jan.-March 2021, doi: 10.1109/MMUL.2020.3034338.

[24] Peining Zhen, Hai-Bao Chen, Yuan Cheng, et al., Fast Video Facial Expression Recognition by a Deeply Tensor-Compressed LSTM Neural Network for Mobile Devices. Association for Computing Machinery. November 2021, Article No.: 23, pp 1-26. https://doi.org/10.1145/3464941.

\section{Biographies}

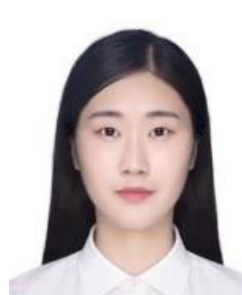

Xue Li Chinese from Weinan City, Shaanxi Province . Born in January 1997. A master's degree candidate from Xidian University. Research directions: machine learning, image processing, cloud security, etc. 